\title{
Dispersion of natural modes in a many-layer planar system
}

\author{
A.V. Goncharenko, B.A. Snopok, Yu.M. Shirshov, E.F. Venger, S.N. Zavadskii \\ Institute of Semiconductor Physics, NAS Ukraine, 45 Prospect Nauki, Kyiv, 03028, Ukraine \\ Tel.: (380-44) 265-60-20; E-mail: avg@isp.kiev.ua
}

\begin{abstract}
We derive the recurrence dispersion equations for natural modes of a many-layer planar system. As an illustration a five-layer planar system is considered, and solutions for guided-wave polaritons of such a system are found. The behavior and peculiarities of these solutions are analyzed.
\end{abstract}

Keywords: dispersion equation, guided wave polariton, planar system.

Paper received 10.02.00; revised manuscript received 05.07.00; accepted for publication 12.07.00.

\section{Introduction}

It is well known that localized modes of two types may exist in planar systems without spatial dispersion. These modes are often referred to as surface and guided-wave polaritons $[1,2]$. Both of them are non-radiative excitations, natural modes of a system; they represent particular solutions to the wave equation. However, they essentially differ in their behavior. Within the linear optics approximation the surface modes can be $p$-polarized only; they are localized at the boundary between media. Contrary to this, the guided-wave modes may be either $p$ - or $s$ polarized; they are localized within one or several layers.

Many authors have comprehensively studied dispersion of natural modes in planar systems. In [3-10] the corresponding dispersion equations were considered and behavior of their solutions for three- and four-layer systems was analyzed. In general one may believe that these cases have been investigated rather thoroughly. But when the number of layers is over four, then numerical methods are used to calculate the dispersion curves for natural modes. These methods are commonly reduced to a direct solution of the wave equation with defined boundary conditions [11]. There exists also an analytical approach. It leads to a matrix equation [12]. The latter, however, seems to be too cumbersome.

One should particularly mention spatially-nonuniform insulating media $[13,14]$ and multilayer periodic systems (superlattices) [15-17]. The first of them may be treated as a generalization of a planar multilayer system, while the latter may be considered as an important special case. It should be noted that the dispersion equation for natural modes of an arbitrary planar heterosystem is similar (within the phenomenological treatment) to the Schrödinger equation for wave functions that determine a spectrum of energy states in systems with size quantization [18].

Here we get the dispersion relations for natural TE and TM modes of an arbitrary $n$-layer system. These relations are obtained by analytically solving the wave equation. The solutions are rather simple and convenient for practical applications. As an illustration we calculate the dispersion curves for TE and TM guided-wave modes of a five-layer system that is realized in the planar polarization interferometer. We also demonstrate how the spatial distribution of the field $E$ behaves in such a system when frequency changes.

\section{Formalism}

Natural modes are obtained by solving a characteristic equation to which the Maxwell equations are reduced. The Maxwell equations satisfy the boundary conditions that are determined by the problem geometry. By transforming the Maxwell equations (and using also the socalled material equations $D=\varepsilon E$ and $B=\mu H$ ) one can obtain the differential equations for the fields $\vec{E}$ and $\vec{H}$. Generally the wave equations are written as

$$
\nabla^{2} E-\mu \varepsilon \frac{\partial^{2} E}{\partial t^{2}}+(\nabla \ln \mu) \times(\nabla \times E)+\nabla(E \nabla \ln \varepsilon)=0
$$




\section{A.V. Goncharenko et al.: Dispersion of natural modes in a many-layer planar system}

and

$$
\nabla^{2} H-\mu \varepsilon \frac{\partial^{2} H}{\partial t^{2}}+(\nabla \ln \varepsilon) \times(\nabla \times H)+\nabla(H \nabla \ln \mu)=0
$$

For uniform and isotropic media both $\ln \varepsilon$ and $\ln \mu$ vanish and the wave equations (1) and (2) take the following form:

$$
\nabla^{2} E-\mu \varepsilon \frac{\partial^{2} E}{\partial t^{2}}=0, \quad \nabla^{2} H-\mu \varepsilon \frac{\partial^{2} H}{\partial t^{2}}=0 .
$$

These are the traditional wave equations for electromagnetic field. They are satisfied by the well-known solutions in the form of plane waves $\psi=\exp [i(\omega t-k r)]$. Here $\psi$ is considered to mean any of Cartesian components of the vectors $E$ and $H$, and the relationship between the circular frequency $\omega$ and wave vector $k$ is $|k|=\omega \sqrt{\mu \varepsilon}$.

Plane waves are not the only solutions to the wave equations. Another solution is given by the so-called "Gaussian sheaves" that represent the solutions of cylindrical symmetry. For spherically-symmetric problems one obtains solutions in the form of spherical waves.

Let us consider a two-dimensional medium involving $n$ layers (Fig. 1). Every $i$-th layer (whose thickness is $d_{i}$ ) is characterized by its complex permittivity $\varepsilon_{i}$. The first and last layers are semiinfinite. All the layers are assumed to be magnetically non-active $\left(\mu_{i}=1\right)$, uniform and isotropic. To obtain the solution to equations (3) in such a media, one has to sew the solutions for individual layers together at the corresponding boundaries. When solving the above problem, it is convenient to consider solutions to wave equations for vector components $E_{y}$ и $H_{y}$. Let us consider a solution for the field with vector $E$ component along the $y$-axis. We shall seek this solution in the form of plane

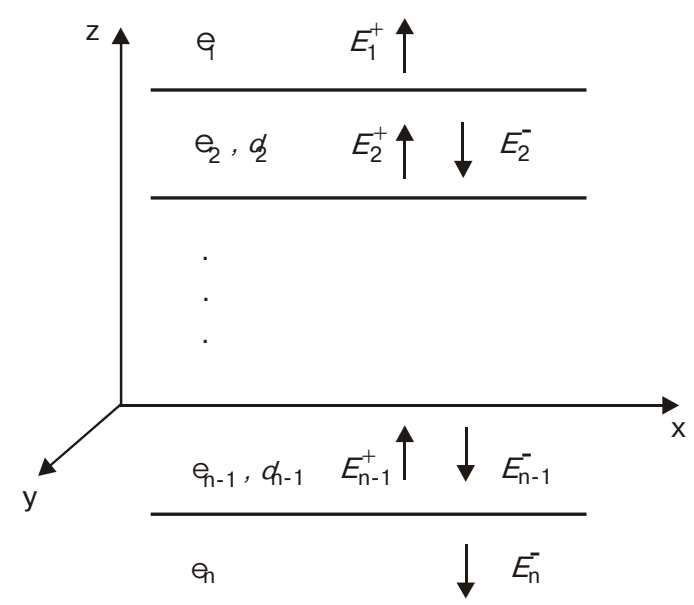

Fig. 1. A diagram of the $n$-layer planar system considered. waves, so $E=\left(0, E(z) \exp \left[i\left(\omega t-k_{x} x\right)\right], 0\right)$. One gets for every layer:

$\left[\frac{\partial^{2}}{\partial z^{2}}+\left(k_{0}^{2} n_{j}^{2}-k_{x}^{2}\right)\right] E(z)=0, j=1, n$.

The guided-wave (localized) solutions in the first and last layers involve the outgoing waves only. Let us write down the mode function and obtain a dispersion relation for the existing types of waves:

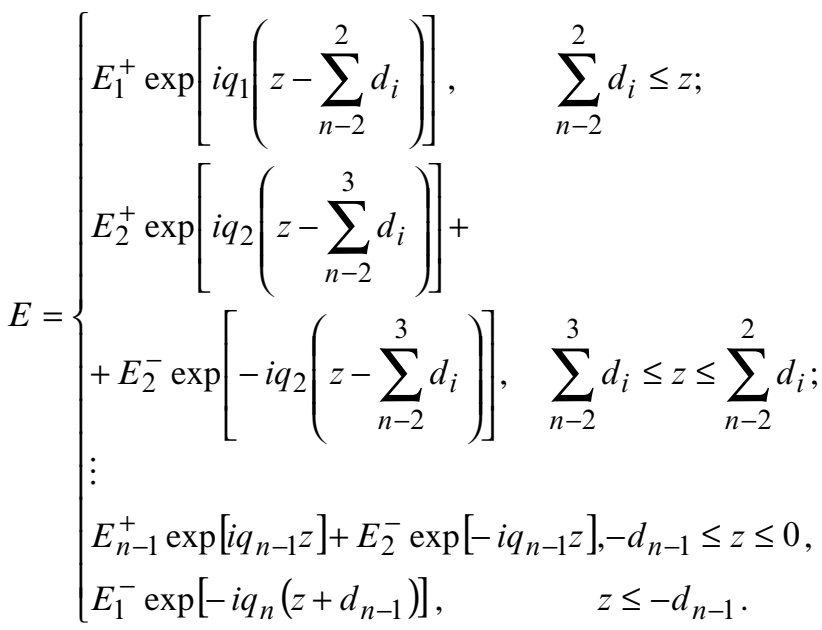

For the (n-1)-th layer one can present the electric field as

$\tilde{E}_{n-1}^{+}\left[\cos \left(q_{n-1} z\right)+i \frac{1-R_{n-1, n-2}^{s}}{1+R_{n-1, n-2}^{s}} \sin \left(q_{n-1} z\right)\right]$

where $\tilde{E}_{n-1}^{+}=E_{n-1}^{+} /\left(1+R_{n-1, n-2}^{s}\right)$ and $R_{n-1, n-2}^{s}$ is the amplitude coefficient of TE-wave reflection at the boundary between the (n-1)-th and (n-2)-th layers. It should be noted that both $R_{n-1, n-2}^{S}$ and $R_{n-1, n-2}^{p}$ (the reflection coefficient for TH-wave) may be easily calculated using the standard recurrence relations for reflection from a multilayer medium (see, e.g., [19]).

The field in the $n$-th layer is:

$$
\begin{aligned}
& \tilde{E}_{n-1}^{+}\left[\cos \left(q_{n-1} d_{n-1}\right)-i \frac{1-R_{n-1, n-2}^{s}}{1+R_{n-1, n-2}^{s}} \sin \left(q_{n-1} d_{n-1}\right)\right] \times \\
& \times \exp \left[-i q_{n}\left(z+d_{n-1}\right)\right]
\end{aligned}
$$

Imposing the continuity condition on the field derivatives at the boundary between the $n$-th and ( $n$-1)-th layers, one gets the dispersion relation for a multilayer planar system: 


\section{A.V. Goncharenko et al.: Dispersion of natural modes in a many-layer planar system}

$$
\tan \left(q_{n-1} d_{n-1}\right)=-i \frac{\frac{1-R_{n-1, n-2}^{s}}{1+R_{n-1, n-2}^{s}}+\frac{q_{n}}{q_{n-1}}}{1+\frac{q_{n}\left(1-R_{n-1, n-2}^{s}\right)}{q_{n-1}\left(1+R_{n-1, n-2}^{s}\right)}}
$$

After generalization to an arbitrary $(j$-th) layer one obtains:

$$
\begin{aligned}
& \tan \left(q_{j} d_{j}\right)=-i \frac{\frac{1-R_{j, j-1}^{s}}{1+R_{j, j-1}^{s}}+\frac{1-R_{j, j+1}^{s}}{1+R_{j, j+1}^{s}}}{1+\frac{\left(1-R_{j, j-1}^{s}\right)\left(1-R_{j, j+1}^{s}\right)}{\left(1+R_{j, j-1}^{s}\right)\left(1+R_{j, j+1}^{s}\right)}} \cdot \\
& \text { The expression } \frac{1-R_{j, j-1}^{s}}{1+R_{j, j-1}^{s}} \text { determines the amplitude- }
\end{aligned}
$$
phase characteristics at the boundary between the $j$-th and (j-1)-th layers. If $\left|R_{j, j-1}^{s}\right|=1$, then $\frac{1-R_{j, j-1}^{S}}{1+R_{j, j-1}^{S}}=i \tan \left(\frac{\varphi_{j, j-1}}{2}\right) ;$ for the case of a boundary between two media in the total internal reflection region $\tan \left(\frac{\varphi_{2,1}}{2}\right)=-i \frac{q_{1}}{q_{2}}$. For a three-layer planar waveguide one gets the following well-known expression:

$\tan \left(q_{2} d\right)=i \frac{q_{2}\left(q_{1}+q_{3}\right)}{q_{2}^{2}+q_{1} q_{3}}$.

It is often written down as

$q_{2} d=\arctan \left(\frac{h_{1}}{q_{2}}\right)+\arctan \left(\frac{h_{3}}{q_{2}}\right)+m \pi$,

where $m$ is the mode number and (as was noted before) $\frac{h_{1}}{q_{2}}=\tan \left(\frac{\varphi_{1,2}}{2}\right)$ and $\frac{h_{3}}{q_{2}}=\tan \left(\frac{\varphi_{1,3}}{2}\right)$.

When calculating the excited modes for a composite planar system with a ( $j$-th) guided-wave layer at whose boundaries $|R|=1$, it is practically convenient to use the following expression:

$q_{j} d_{j}=\frac{\varphi_{j, j-1}}{2}+\frac{\varphi_{j, j+1}}{2}+m \pi$

where $m$ is an integer.

The above consideration dealt with the TE-mode propagation in planar systems. For TM-modes an ap- proach is the same. After sewing the tangential field components, $H_{y}$ and $E_{x}$, together at the media boundaries one obtains the following dispersion relation for the TM-mode:

$$
\tan \left(q_{j} d_{j}\right)=-i \frac{\frac{1-R_{j, j-1}^{p}}{1+R_{j, j-1}^{p}}+\frac{1-R_{j, j+1}^{p}}{1+R_{j, j+1}^{p}}}{1+\frac{\left(1-R_{j, j-1}^{p}\right)\left(1-R_{j, j+1}^{p}\right)}{\left(1+R_{j, j-1}^{p}\right)\left(1+R_{j, j+1}^{p}\right)}} .
$$

Expressions (9) and (13) may be obtained from each other through changing from $R^{p}$ to $R^{S}$ and vice versa. For two types of mode expression (13) is written down similarly. In one case by $\varphi_{j, j-1}$ is meant the phase shift for TE-waves, while in another case the phase shifts for the incident and reflected $\mathrm{TH}$-waves are meant. The analogs to expressions (10) and (11) are, respectively, the following expressions:

$\tan \left(q_{2} d\right)=i q_{2} \frac{q_{1} \frac{\varepsilon_{2}}{\varepsilon_{1}}+q_{3} \frac{\varepsilon_{2}}{\varepsilon_{3}}}{q_{2}^{2}+q_{1} q_{3} \frac{\varepsilon_{2}}{\varepsilon_{1}} \frac{\varepsilon_{2}}{\varepsilon_{3}}}$

and

$q_{2} d=\arctan \left(\frac{h_{1}}{q_{2}} \frac{\varepsilon_{2}}{\varepsilon_{1}}\right)+\arctan \left(\frac{h_{3}}{q_{2}} \frac{\varepsilon_{2}}{\varepsilon_{3}}\right)+m \pi$.

\section{Some features of mode structure for a five- layer system}

To illustrate, let us consider a five-layer planar structure forming the main element of a planar polarization interferometer that is in contact with water $[20,21]$. The layer parameters in the region of He-Ne laser generation are: $n_{1}=1.33, k_{1}=0$ (water); $n_{2}=1.51, k_{2}=0, d_{2}=1.2 \mu \mathrm{m}$ (protective layer made from phosphosilicate glass); $n_{3}=2$, $k_{3}=0, d_{3}=190 \mathrm{~nm}$ (guided-wave layer made from silicon nitride); $n_{4}=1.49, k_{4}=0, d_{4}=1.2 \mu \mathrm{m}$ (silicon oxide interlayer); $n_{5}=3.83, k_{5}=0.02$ (silicon plate).

Modes of different types may be excited between the media 1 and 5 in such a system. One can see that the medium 5 has the highest refractive index. Solutions to the wave equation in this medium will be always harmonic functions. One may, however, expect light localization, i.e., the guided-wave propagation of light here. Those modes whose energy is localized in the silicon nitride layer will tunnel to the substrate only slightly. The modes that are localized in the silicon nitride and protective layers are also weakly radiative. But if energy is localized within the three inner layers, then these modes will demonstrate some radiation losses due to absence of total internal reflection at the boundary between silicon oxide and silicon. Such modes may be named leak modes. When $k_{x}>k_{z}$, then these modes may slightly radiate. 


\section{A.V. Goncharenko et al.: Dispersion of natural modes in a many-layer planar system}

Shown in Fig. 2 is the solution to dispersion equation for the above system. The dashed curves correspond to the so-called light straights in media that form the system considered $\left(v_{i}=k_{x} / 2 p n_{i}\right.$, where $\left.v=\omega / 2 p c\right)$. The dispersion relations were calculated from expressions (11) and (15). The full (dashed) curves determine TH- (TE-) mode dispersion. One can see that at high frequencies the dispersion curves are close to the light straight 3 that corresponds to light propagation in silicon nitride. When the frequency goes down, then the dispersion curves begin to meet the light straights that correspond to light propagation in media with lower refractive index (dashed curves $2,4,1)$. Between the curves 3 and 2 the mode field is localized in the guided-wave layer, while in the neighboring layers the fields die out exponentially. When frequency decreases, then the wave cannot go into this layer and becomes delocalized. Between the light straights 2 and 4 the solutions are given by harmonic functions in the silicon nitride and phosphosilicate glass layers, while in water and silicon oxide the fields die out exponentially. At the intersection of dispersion curves and the light straight 4 in silicon oxide, the fields become harmonic too. Since no total internal reflection occurs at the silicon oxide-silicon interface, these oscillations demonstrate high radiation losses.

A section of dispersion relations in the $\mathrm{He}-\mathrm{Ne}$ laser generation region is shown in Fig. 2 on a larger scale. At the laser generation frequency (horizontal dashed line $\left.v=15803 \mathrm{~cm}^{-1}\right)$ a substantial $\left(k_{x}^{T E}-k_{x}^{T H} \approx 8 \cdot 10^{4} \mathrm{~cm}^{-1}\right)$ difference between the propagation constants for waves of different polarization exists for zero-order modes only. Even for the first-order mode $k_{x}^{T E}-k_{x}^{T H} \approx 50 \mathrm{~cm}^{-1}$, i.e., this difference is very small, and the corresponding curves in Fig. 2 merge together. There are only two pairs of modes with low damping at this frequency. Other solutions (four pairs) are the outgoing modes. They are characterized by high radiation losses.

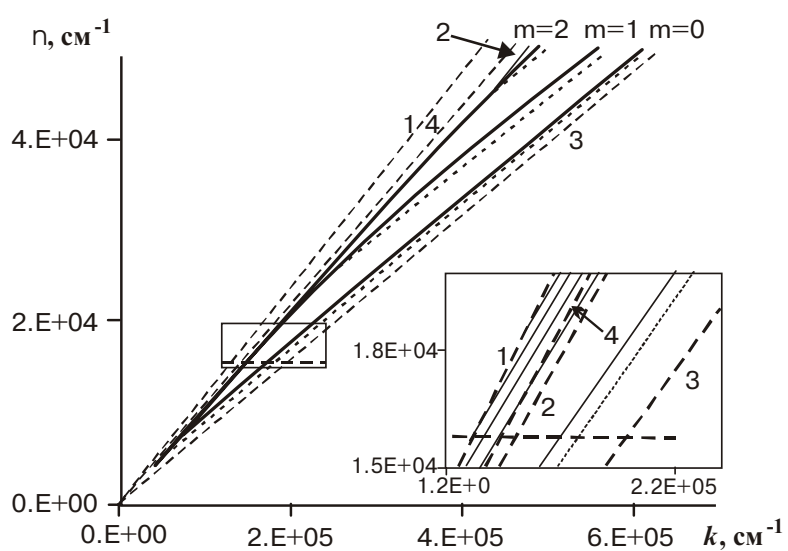

Fig. 2. The guided-wave mode dispersion curves for a fivelayer system. (inset - a fragment of the same curves on an enlarged scale in the region of $\mathrm{He}-\mathrm{Ne}$ laser generation).
In practical applications all the modes but that of zero order are undesirable. Therefore choice of layer parameters is of essence. Thus at thick silicon oxide layer one may get rather low radiation losses for the leak modes. And this is unwanted when using planar polarization interferometer.

To picture the behavior of localized modes in more detail, we have calculated their intensities, $I \propto|E|^{2}$, at different points by numerically solving the wave equation (4). Shown in Fig. 3 are the lower-order modes that may be excited in the planar system considered. We have calculated the field intensity distributions for TE-modes at a frequency of the He-Ne laser generation (about $\left.15800 \mathrm{~cm}^{-1}\right)$. The layer boundaries are shown with vertical dashed lines. For zero $(m=0)$ mode the field is localized to a large extent within the guided-wave layer. Some portion of light that propagates beyond the guided-wave layer may interact with adsorbed molecules. This leads to a change in the signal phase. For TH-modes the fields are localized to a lesser extent (their localization depth is bigger), so they interact with adsorbates more strongly.

A feature of the mode structure in a composite planar system is absence of symmetry in the field spatial distributions similar to those presented in Fig. 3. It seems convenient to classify modes (as in the simplest cases) according to number of field intensity minima in the inner layers. A form of field intensity distribution for a mode of some order may strongly vary when the dispersion curve intersects the light straights in different media. To illustrate, one can see from Fig. 4 that the first-order mode is spreading (i.e., its energy is flowing) from the guided-wave layer to the protective layer when frequency goes down. In this case a limiting (critical) point exists that corresponds to a frequency of about $22310 \mathrm{~cm}^{-1}$. At this point a transition occurs from exponentially damping solutions in the region 2 to harmonic (oscillating) ones. Such critical points exist for all modes. A transition through this point is ac-

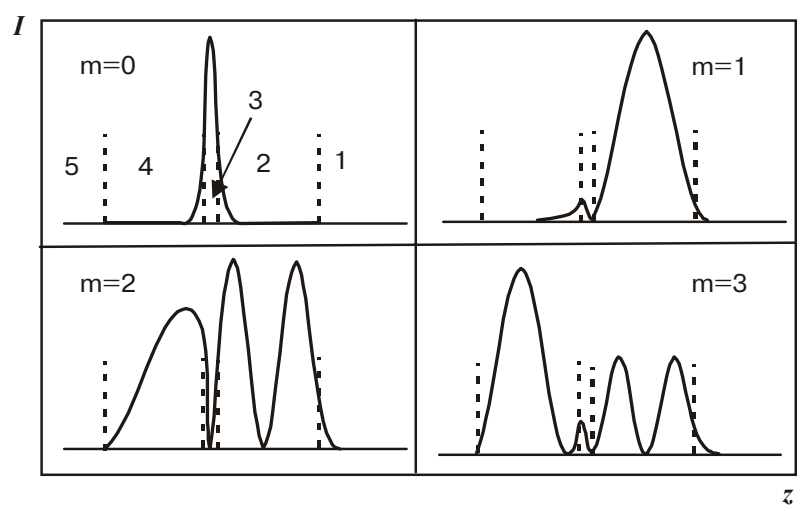

Fig. 3. Field intensity spatial distribution for the guided-wave TE-modes of the first four orders. 


\section{A.V. Goncharenko et al.: Dispersion of natural modes in a many-layer planar system}

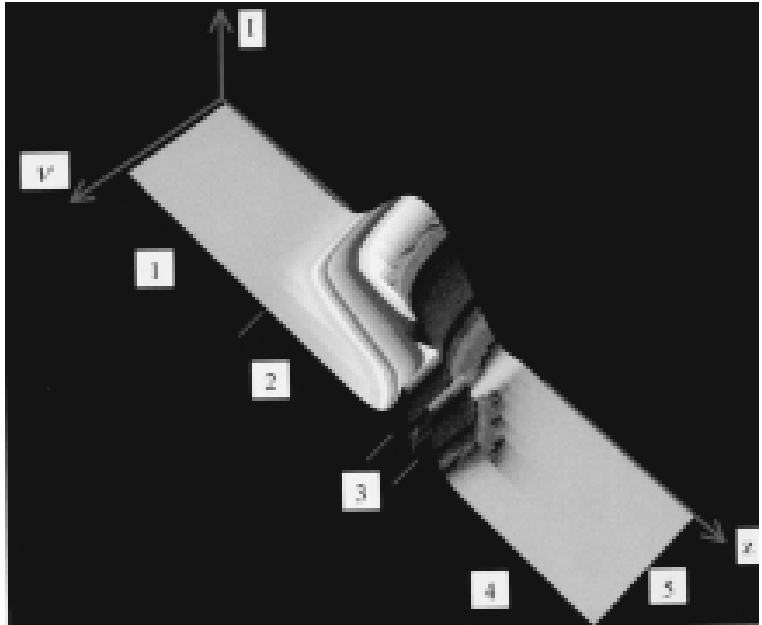

Fig. 4. Natural mode intensities for a five-layer system in $(n, z)$ coordinates.

companied by substantial energy redistribution between the layers 2 and 3, as can be seen from the results presented.

In conclusion we would like to note that no surface linear localized waves can propagate in the system considered, since for their existence it is necessary that $\operatorname{Re} \varepsilon_{j}<0$ at least for one of the layers [2]. Obviously nonlinear surface waves may exist here, but to describe them one should use a nonlinear dispersion equation [22].

\section{Conclusion}

By solving the wave equation we have got dispersion relations for TE and TH natural (localized) modes in a multilayer planar system with arbitrary number of layers. The obtained relations are convenient for practical applications. They enable one to find rather easily the $v(k)$ dependencies for all possible types of natural modes by using standard recurrence relations for reflection coefficients of a multilayer system. As an example we considered a five-layer system whose natural modes are guided-wave polaritons. We have found all possible solutions to dispersion equations for this system and performed an analysis of their behavior.

\section{References}

1. A. Yariv, P. Yeh, Optical Waves in Crystals, Wiley, New York (1984)

2. Surface Polaritons, Eds. V.M. Agranovich, D.L. Mills, Amsterdam, North-Holland Publ. Co. (1982).
3. R. Fuchs, K.L. Kliever, Optical modes of vibration in an ionic crystal slab // Phys. Rev. 140, pp. A2076-A2088 (1965).

4. K.L. Kliever, R. Fuchs, Optical modes of vibration in an ionic crystal slab including retardation. I. Nonradiative region // Phys. Rev. 144, pp. $495-503$ (1966).

5. K.L. Kliever, R. Fuchs, Optical modes of vibration in an ionic crystal slab including retardation. II. Radiative region // Phys. Rev. 150, pp. 573-586 (1966).

6. L. Wendler, E. Jager, Phonon-polariton in bilayer systems. Surface phonon-polaritons // Phys. stat. sol. (b) 120, pp. 235247 (1983).

7. L. Wendler, Phonon-polaritons in bilayer systems. $S$-polarized guided wave phonon-polaritons // Phys. stat. sol. (b) 128, pp. 425-437 (1985).

8. V.V. Bryksin, Yu.M. Gerbshtein, D.N. Mirlin, Surface optical vibrations in ionic crystal slabs (in Russian) // Fiz. Tverd. Tela 14(2), pp. 543-552 (1972).

9. I.I. Burshta, E.F. Venger, Yu.A. Pasechnik, O.V. Snitko, Simplest properties of guided wave polaritons in three-layer structures // Phys. stat. sol. (b) 146, pp. 517-524 (1988).

10. E.F. Venger, A.V. Goncharenko, S.N. Zavadskii, Dispersion equation for surface polaritons in a thin film (in Russian) // Pis'ma v ZhTF 18(5), pp. 64-68 (1992).

11. G.E. Smith, M. Hill, Phase matching in four-layer optical waveguides // IEEE J. Quant. Electron. VQE-4(5), pp. 288289 (1968).

12. O.S. Gorya, E.P. Pokatilov, Collective excitations in multilayer structures (in Russian) // Deposited in Moldavian Scientific \& Research Institute of Scientific \& Technical Information, registration N 541M-D85, Kishinev (1985).

13. E. Conwell, "Guided" polaritons and plasmons in media with surface inhomogeneities // Solid State Commun. 14, pp. 915-917 (1974)

14. M.L. Dmytruk, Yu.V. Kryuchenko, Dispersion of surface plasmon polaritons in spatially-nonuniform transition layers (in Ukrainian) // Ukrainskii Fiz. Zhurn. 35(1), pp. 57-64 (1990).

15. A. Caille, M. Banville, M.J. Zuckermann, Interaction of interface plasmons and longitudinal optical phonons in a multilayer structure // Solid State Commun. 24, pp. 805-808 (1979).

16. I.V. Kozhevnikov, On surface electromagnetic waves in superlattices (in Russian) // Poverknost' 9, pp. 26-32 (1986).

17. E.L. Ivchenko, V.A. Kosobukin, Excitonic polaritons in semiconductors with a superlattice (in Russian) // Fiz. Tekhn. Poluprov. 22(1), pp. 24-30 (1988).

18. O.S. Gorya, Polaritons in layered structures and dimensional quantization (in Russian) // Fiz. Tverd. Tela 38(11), pp. 34553460 (1996).

19. G.V. Rosenberg, Optics of Thin-Film Coatings (in Russian), Fizmatgiz, Moscow (1958).

20. Yu.M. Shirshov, S.V. Svechnikov, A.P. Kiyanovskii, Yu.V. Ushenin, E.F. Venger, R. Merker, A sensor based on the planar-polarization interferometer // Sensors and Actuators A68, pp. 384-387 (1998).

21. E.F. Venger, A.V. Goncharenko, S.N. Zavadskii, B.A. Snopok, Yu.V. Ushenin, Yu.M. Shirshov, Numerical simulation of the effect of coating film thickness and molecular adsorption on propagation angle in a planar polarization interferometer (in Russian) // Optoelektronika i Poluprovodnikovaya Tekhnika 34, pp. 142-148 (1999).

22. N.L. Dmitruk, V.G. Litovchenko, V.L. Strizhevskii, Surface Polaritons in Semiconductors and Insulators (in Russian), Naukova Dumka, Kiev (1989). 